\title{
Schwannomas of the upper extremity: analysis of 34 cases
}

\author{
Roberto Adani • Luigi Tarallo • Raffaele Mugnai • \\ Stefano Colopi
}

Received: 11 May 2014 / Accepted: 1 September 2014

(C) Springer-Verlag Wien 2014

\begin{abstract}
Background Schwannomas are the most common benign tumours developing in peripheral nerves. They usually present as a slow-growing mass, sometimes associated with pain and paraesthesia. The aim of this study is to define the correct preoperative diagnosis, to review the surgical treatment employed and to evaluate short- and long-term neurological deficits.

Methods Thirty-four patients affected by schwannoma in the upper limbs were treated in the period 1995-2011. In 15 patients the tumour was located on the ulnar nerve, in 8 on the median nerve, in 2 on the radial nerve, in 1 on the anterior interosseous nerve, in 1 on the muscle-cutaneous nerve, and in the remaining 7 on the digital nerves. All patients were surgically treated using a microsurgical approach.

Results The enucleation of the mass was possible without fascicle lesion in 12 cases. In 22 cases resection of the indissociable fascicles was performed. Postoperative paraesthesia was present in 28 out of 34 treated patients; this clinical sign regressed in a mean period of 12 months in 27 patients.

Conclusions When approaching a palpable mass in the upper limbs, the possibility of a peripheral nerve tumour should always be considered. It is important to look for typical signs of schwannomas, such as a positive Tinel sign and peripheral paraesthesia. Imaging assessment with magnetic resonance
\end{abstract}

R. Adani $(\bowtie)$

Department of Hand Surgery and Microsurgery, University Hospital Verona, Ospedale GB Rossi, Piazzale LA Scuro 10, Verona, Italy

e-mail: roberto.adani@ospedaleuniverona.it

L. Tarallo $\cdot$ R. Mugnai

Department of Orthopedic Surgery, University of Modena and

Reggio Emilia, Modena, Italy

S. Colopi

Department of Radiology, University of Modena and Reggio Emilia, Modena, Italy imaging (MRI) and ultrasonography enables the determination of where the tumour takes its origin and from which nerve. Microsurgical techniques and know-how are recommended in approaching the resection in order to respect as many nerve fibres as possible.

Keywords Schwannoma $\cdot$ Neurilemmoma $\cdot$ Peripheral nerve tumours $\cdot$ Magnetic resonance imaging $\cdot$ Microsurgery .

Enucleation

\section{Introduction}

Schwannoma is a benign tumour developing from Schwann cells. In $95 \%$ of cases schwannomas are usually solitary [27], but multiple tumours have also been reported [20, 28, 29]. They are characterised by a slow non-infiltrating pattern of growth for several years without any specific symptoms [8]. The incidence of schwannomas in eastern countries is $5 \%$ in adults and $2 \%$ in children [5]. Because of this low incidence, the clinical signs and symptoms are often misunderstood, somehow associated to other soft tissue tumours such as ganglions, tenosynovitis or other soft-tissue tumours [26]. The tumour is often associated with a "tingling sensation", which is not constant at clinical evaluation. Hems et al. [6] reviewed a series of 104 peripheral-nerve benign tumours and demonstrated that in only seven cases was the preoperative diagnosis accurate. Schwannomas are well encapsulated and surgical enucleation is generally believed to be routinely possible without causing neurological deficit [12, 21, 25]. This statement is not always true as reported by other authors $[17,24]$. The purpose of this retrospective study of 34 schwannomas of the upper limb treated in a period of 16 years was to define the correct preoperative diagnosis, to review the surgical treatment employed and to evaluate short- and longterm neurological deficits. 


\section{Materials and methods}

Thirty-four schwannomas located in the upper extremity were treated between January 1995 and December 2011. The study included only histopathologically confirmed schwannomas of the peripheral nerves of the upper limb; patients who had undergone previous surgery or open biopsy were excluded. A patient with multiple schwannomas and one with schwannomas in different nerves were not included. Institutional review board approval was obtained for medical chart review and clinical follow-up. The clinical and pathological medical records were also reviewed. Data included age, sex, location, symptoms, preoperative investigations, histological diagnosis and clinical follow-up. There were 19 men and 15 women; the mean age at the time of operation was 44 (range, 20-78) years. The time between onset of symptoms and surgical excision ranged from 4 to 84 (mean 32) months. There were 15 schwannomas originating from the ulnar nerve, 8 from the median nerve, 2 from the radial nerve, 1 from the anterior interosseous nerve, 1 from the muscle-cutaneous nerve and 7 from the digital nerves. Topographically, 7 were localised in the arm, 14 in the elbow-forearm region and 13 in the wrist-hand region. A palpable mass was present in 32 cases (94\%), 14 patients had peripheral nerve symptoms ( $41 \%$ ) (such as paraesthesia to the territory of the affected nerve) and 23 presented a positive Tinel sign (68\%). No patient had motor weakness. Spontaneous pain was present in 12 cases (35\%). In 32 cases a magnetic resonance imaging (MRI; $94 \%$ ) scan was performed to study tumour dimension, anatomical compartment involved, detectability of the nerve origin, relationship with the mass, signal intensity on T1- and T2-weighted images and the presence of a capsule. Ultrasound imaging was used in 11 cases. Standard biopsy or fine-needle aspiration were never performed.

All patients were treated with a microsurgical approach under loupe magnification or using the microscope by the senior author (R.A.). A longitudinal incision was done over the tumour and the epineurium. The tumour capsule was incised and the shiny surface of the tumour was exposed; the uninvolved nerve fibres were isolated and then retracted. An interfascicular dissection was performed with isolation of the nerve fibres entering and exiting the mass; finally, the tumour was enucleated (Fig. 1) in one piece without involvement the surrounding nerve fascicles. If a nerve fascicle was entrapped into the mass at the proximal and distal poles, we tried to isolate it from the tumour using meticulous microsurgical dissection (Fig. 2); when this was not possible, the fascicle had to be resected together with the tumour (Fig. 3). We never performed an intraoperative nerve conduction study to identify non-functioning fascicles.

Clinical follow-up was at 1 or 2 weeks, at medium term (between 3 and 6 months) and at 1 year. The neurological status of each patient was evaluated considering: pain, paraesthesia and weakness.

\section{Results}

The histology of the excised specimens showed the typical characteristics of schwannomas in 29 cases; five cases were diagnosed as ancient schwannoma. A correct preoperative diagnosis was possible in $29(85 \%)$ out of 34 treated patients (misdiagnosis cases: two ganglia, one neurofibroma, one giant-cell tumour of the tendon sheath and one was not precisely diagnosed). MRI was performed in 32 cases, and in 29 $(91 \%)$ the schwannomas were correctly diagnosed. Misdiagnosis cases: one ganglion, one neurofibroma and one not precisely diagnosed. All tumours showed the typical aspect of schwannomas: moderately bright in proton-densityweighted images and bright in T2-weight images; four cases were diagnosed as ancient schwannoma. In 21 schwannomas a probable capsule was identified and this feature appeared as a low-density area; in 28 patients the nerve was in an eccentric position with respect to the mass.

The enucleation of the mass was possible without fascicle damage in 12 cases ( $35 \%$ ), while in 22 cases (65\%) resection of the indissociable fascicles was performed.

Sensory deficit was recognised immediately after surgery in $28(82 \%)$ out of 34 treated patients; 14 out of 20 patients who had no subjective symptoms preoperatively developed hypoaesthesia or paraesthesia postoperatively. This impairment was tolerable and regressed in a mean period of 12 months in 27 patients. Only one patient reported a debilitating neuropathic pain 1 year after excision of a schwannoma from the ulnar nerve located in the elbow region. No other patients reported major neurological deficit after surgery; none had motor palsy or severe weakness.

Location of the tumour and affected nerve (except for the digital nerves in which all the cases were characterised by postoperative sensory deficit) did not appear to modify the final outcome. Also, the capability to reach complete enucleation of the schwannoma preserving all the fascicles (12 cases: 6 schwannomas originating from the ulnar nerve, 4 from the median nerve, 1 from the radial nerve and 1 from the digital nerve) or including one of the normal fascicles (22 cases: 9 schwannomas originating from the ulnar nerve, 4 from the median nerve, 1 from the radial nerve, 1 from the anterior interosseous nerve, 1 from the muscle-cutaneous nerve and 6 from the digital nerves) did not affect the postoperative outcome.

There was no evidence of any schwannoma recurring during the follow-up period.

\section{Discussion}

In the treatment of schwannomas a correct diagnosis is the first essential step [19, 24]. Preoperative identification is difficult and the risk of misdiagnosis is high because their 
Fig. 1 Left: Schwannoma of the ulnar nerve isolated from the ulnar artery. Right: The tumour was easily shelled out from the nerve with minimal dissection
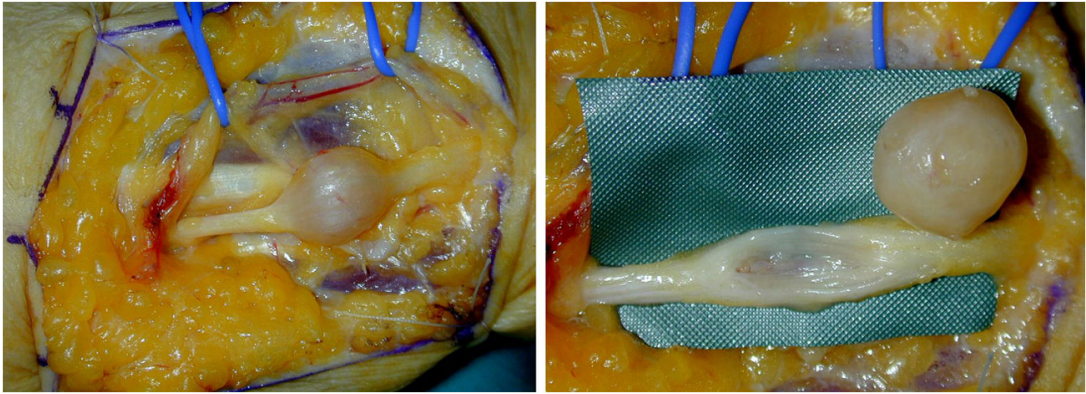

symptoms are not always clear. As reported also by other authors $[11,19]$ the Tinel sign, which was present in $68 \%$ of our patients, is the most useful alert sign in the diagnosis. When it is possible to observe an isolated, palpable slowgrowing mass, usually located on the volar surface of the limb with a positive Tinel sign, a suspected schwannoma should be considered [1]. The diagnosis of a soft tissue tumour is usually confirmed by open biopsy, which is not recommend when a peripheral nerve sheath tumour is presumed due to the high risk of iatrogenic nerve injury [19]; an ultrasound-guided needle biopsy seems to be less invasive but the possibility of provoking a nerve fascicle injury is still present. Among many different diagnostic methods, MRI is considered the most helpful in confirming the diagnosis preoperatively $[1,6,9$, 11, 19, 24]. MRI can demonstrate the tumour origin and its anatomical relationship to the surrounding structures. The MRI aspect of a schwannoma is a homogeneous mass, with a middle-low intensity signal at SE T1-weighted sequences, whereas at T2-weighted sequences it shows a high signal. An ancient schwannoma produces a different image, because of its low-density signal areas (Fig. 3), which are caused by hyalin and fibrosis degeneration [10, 30]. Ancient schwannoma is an uncommon variant of schwannoma with a course typical of a slow-growing neoplasm [1]. This subtype is characterised by extensive degeneration and diffuse hypocellular areas, these changes might occur because it takes a long time for ancient schwannomas to develop [30]. Due to the nuclear atypia, and cystic degeneration, ancient schwannomas might be confused with malignant tumours on histology and imaging, leading to a radical surgical approach. After contrast infusion, schwannomas show a large enhancement (like the neurofibromas), whereas in case of necrotic or cystic areas, or if there is a "target sign", the resulting image is different. The "target sign" in $50 \%$ of cases is due to a central low-intensity signal (fibrous component) and a peripheral high-intensity signal (myxedematous component). It has been reported that MRI does not seem to be always adequate to distinguish between schwannomas and neurofibromas $[6,11]$. Schwannomas are frequently encapsulated whereas neurofibromas are usually not; detection of a capsule which causes a low intensity rim at the margin of the tumour and the presence of the nerve along one side of the mass could help to differentiate schwannomas from neurofibromas [1, 3, 9, 28]. In our study MRI identified correctly 29 cases of schwannomas out of the 32 cases studied; a case was initially misdiagnosed as a presumed neurofibroma, one was not diagnosed at all and the last one was thought to be a ganglion in the Guyon canal. Moreover, in these circumstances assessment employing both MRI and ultrasound imaging may be helpful for cases suggestive of schwannoma $[22,30]$. In the past, ultrasounds were
Fig. 2 Upper: Schwannoma of the radial nerve in the lower third of the arm. Sagittal FSE T1weighted imaging showing low to intermediate signal intensity of the lesion. At FSE T2-weighted imaging, the tumour exhibits high signal enhancement, surrounded by a hypointense capsule. At both ends of the lesion a cord-like structure, in continuity with the tumour, is clearly visible, corresponding to the radial nerve. Lower: A nerve fascicle was entrapped in the mass at the proximal and distal poles. It was preserved using a microsurgical dissection
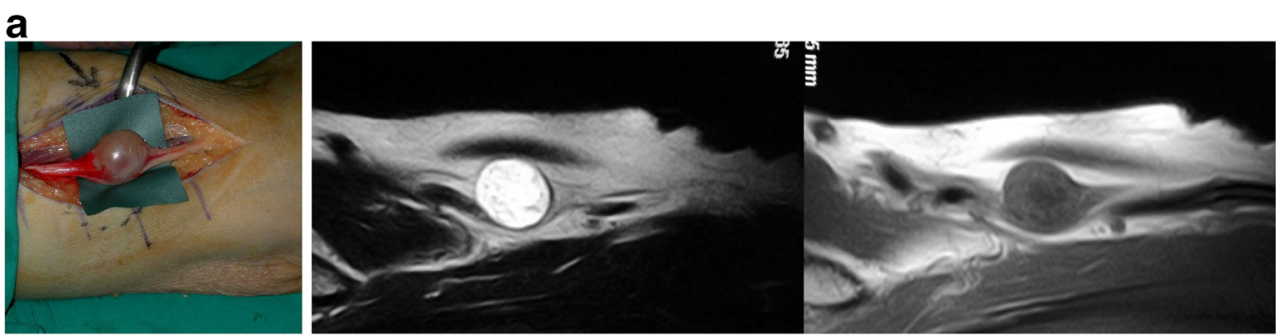

b
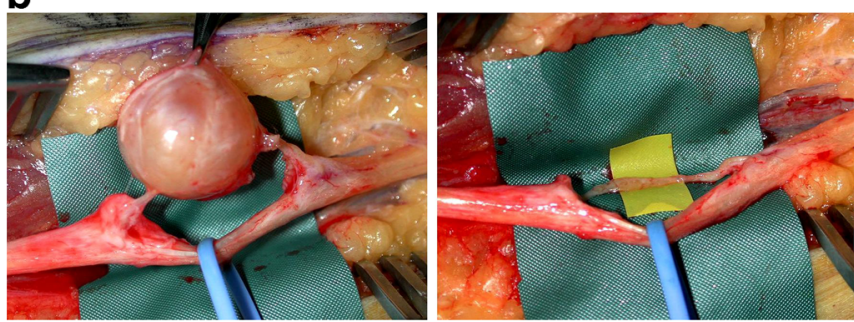
Fig. 3 A palpable mass in the palm. FSE T2-weighed MRI sagittal view showing an ancient schwannoma of the median nerve. The mass has heterogeneous but predominantly high signal intensity and multiple hypointense septa. Nerve fascicle at the proximal and distal poles was entrapped with the tumour and resected

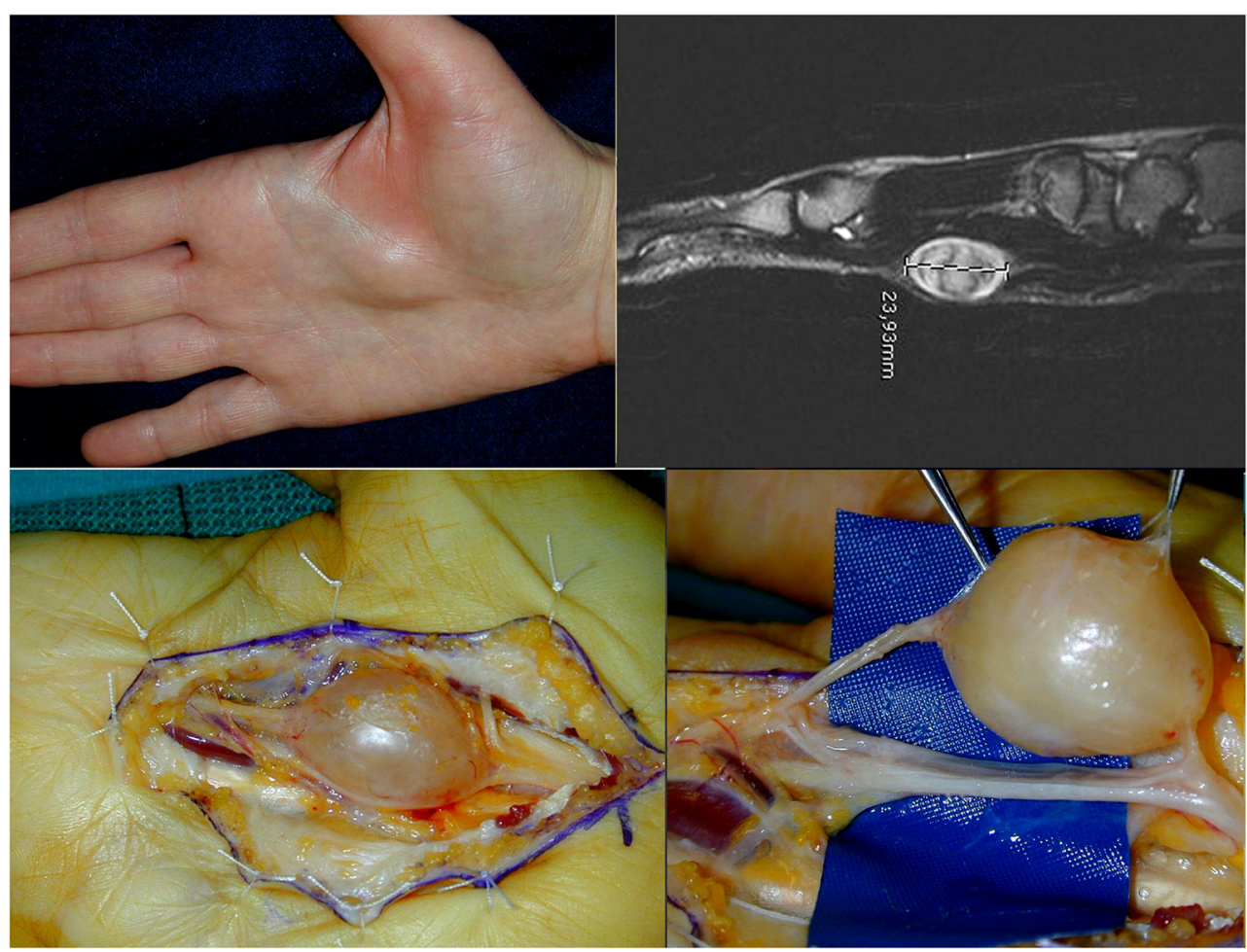

not always able to reveal the connection between the tumour and the nerve, therefore proving to be insufficient to make a correct preoperative diagnosis [7]. Today, recent advances in ultrasound technology provide higher spatial resolution identifying the localisation of the nerve tumour, its origin from a nerve fascicle and its relationship with non-involved nerve
Fig 4 Upper: Axial view at ultrasonography and MRI (FSE T2-weighed) showing the schwannoma $(*)$ under the pronator quadratus muscle $\left(^{\circ}\right)$. Lower: Intraoperative view of the schwannoma under the pronator quadratus muscle connected to the anterior interosseous nerve (AIN; *) a

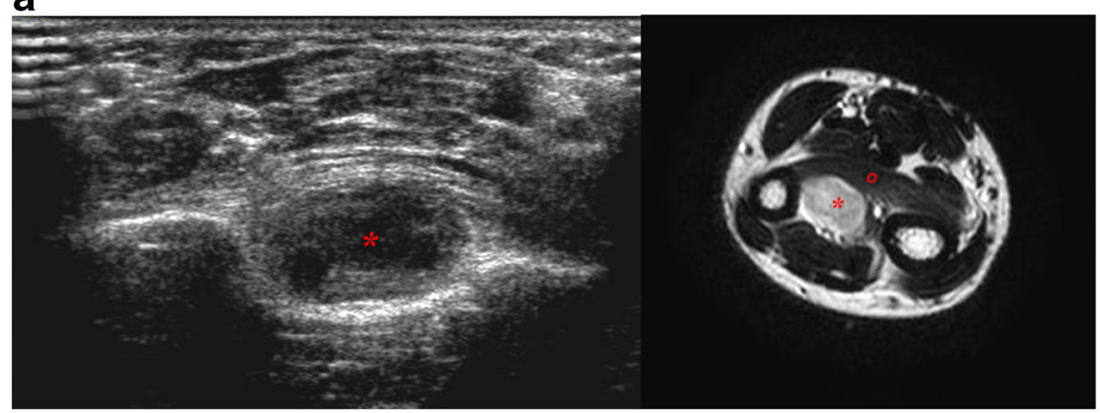

b

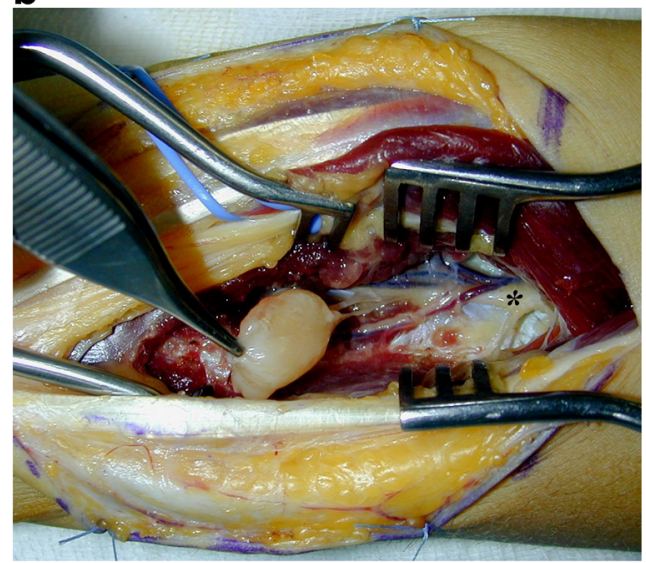


fascicles $[15,16,18]$. Since we were not so familiar with highresolution ultrasound imaging, in this study this technique was used only in few cases, always in combination with MRI (Fig. 4). In our experience MRI provides high diagnostic value for schwannomas with $91 \%$ of correct preoperative diagnoses; this result is superior to those reported by other authors [14, 26], confirming the important role of MRI when approaching nerve tumours $[1,19,28]$.

The elective treatment for schwannomas is enucleation of the tumour from the involved nerve under magnification using microsurgical technique $[1,2,11,13,17-19,24]$. Two main approaches have been reported: extracapsular [11] or intracapsular enucleation [13, 18, 19]. We preferred the intracapsular enucleation because the risk of neural damage seems inferior. Despite this, the enucleation of the tumour without damaging any nerve fascicles was possible only in 12 cases out the 34 cases treated. Repair of the nerve fascicles, resected together with the schwannoma, was never attempted. In a few cases these fascicles were preserved by means of a meticulous microsurgical approach (Fig. 3) but their appearance at the end of the dissection was flat [13] and attenuated [19]. We do not believe, as reported by some authors [19, 24], that resection of one fascicle running through the tumour represents the cause of a major neurological deficit. These types of fascicles are highly nonfunctional, as confirmed by stimulation $[4,13]$. This temporary impairment after surgery was present in most of the cases (82 \%) independently from fascicle preservation. To explain this finding, there are mainly three different reasons [24, 23]. Schwannoma starts from the nerve sheath and the containing fascicle is always involved independently from its residual function; the longitudinal incision, employed during dissection of the tumour, can damage small fascicles; finally, intact fascicles, preserved from the tumour growth, may be compressed during its surgical enucleation resulting in a neuropraxic injury.

In our study, location of the tumour did not appear to modify the final outcome but none of our patients had schwannomas located in the brachial plexus. Sawada et al. [24] reported four cases of schwannoma in the brachial plexus that could not be enucleated completely because many fascicles were involved. In those cases resection of the tumour was performed including some normal fascicles, causing sensory and motor neurological deficits persistent at the final outcome.

All digital schwannomas (seven cases) in our series presented with pain (58\% of all painful cases) as the major complaint, whereas only $18 \%$ of more proximal schwannomas were painful. Moreover, 11 out 12 painful cases were localised in the wrist-hand region. This confirms that pain does not seem to be related to the size of the tumour; rather, to the degree of compression [11,23], the lack of local tissue distensibility and local pressure effects [26], particularly when digital nerves are affected. Microsurgical dissection was performed in all cases and enucleation of the schwannoma preserving all fascicles was possible only in one patient. At 1 week after surgery pain disappeared completely but all the patients reported moderate sensory deficit, which was not reported at medium- and long-term follow-up after 1 year.

Although the schwannoma is cautiously separated from the involved nerve under loupe magnification or under microscope with a microsurgical technique, transient postoperative paraesthesia may still occur; sensibility should recover after a period of a few months to one year in most of the cases, as widely reported in the literature $[1,2,4,8,11,17-19,23,24]$. Sensory recovery in our study occurred in $97 \%$ of the cases at 1-year follow-up. We recommend the combined assessment using both MRI and high-resolution ultrasound imaging, in order to have as many preoperatively correct clinical diagnoses of schwannoma as possible; in this way the patients may be accurately informed of any potential neurological deficits before surgery. Schwannoma can be removed with an acceptable risk of injury to the nerve. High incidence of immediate neurological deficit usually resolves spontaneously.

\section{Conflict of interest None.}

\section{References}

1. Adani R, Baccarani A, Guidi E, Tarallo L (2008) Schwannomas of the upper extremity: diagnosis and treatment. Chir Organi Mov 92: $85-88$

2. Akambi Sanoussi K, Dubert T (2006) Schwannomas of the peripheral nerve in the hand and the upper limb: analysis of 14 cases. Chir Main 25:131-135

3. Beaman FD, Kransdorf MJ, Menke DM (2004) Schwannoma: radiologic-pathologic correlation. Radiographics 24:1477-1481

4. Donner TR, Voorhies RM, Kline DG (1994) Neural sheath tumors of major nerves. J Neurosurg 81:362-373

5. Forthman CL, Blazar PE (2004) Nerve tumors of the hand and upper extremity. Hand Clin 20:233-242

6. Hems TEJ, Burge PD, Wilson DJ (1997) The role of magnetic resonance imaging in the management of peripheral nerve tumors. J Hand Surg [Br] 22:57-60

7. Hoglund M, Muren C, Engkvist O (1997) Ultrasound characteristics of five common soft-tissue tumors in the hand and forearm. Acta Radiol 38:348-354

8. Holdsworth BJ (1985) Nerve tumors in the upper limb-a clinical review. J Hand Surg [Br] 10:236-238

9. Ichikawa J, Sato E, Haro H, Anayama S, Ando T, Hamada Y (2008) Posterior interosseous nerve palsy due to schwannoma: case report. J Hand Surg [Am] 33:1525-1528

10. Isobe K, Tominaga S, Tsutomu A, Hiroyuki K (2004) Imaging of ancient schwannoma. Am J Radiol 183:331-336

11. Kang HJ, Shin SJ, Kang ES (2000) Schwannomas of the upper extremity. J Hand Surg [Br] 25:604-607

12. Kehoe NJ, Reid RP, Semple JC (1995) Solitary benign peripheralnerve tumours. Review of 32 years' experience. J Bone Joint Surg [Br] 77:497-500

13. Kim DH, Murovic JA, Tiel RL, Moes G, Kline DG (2005) A series of 397 peripheral neural sheath tumors: 30 -year experience at Louisiana State University Health Sciences Center. J Neurosurg 102:246-255 
14. Kransdorf MJ (1995) Benign soft-tissue tumors in a large referral population: distribution of specific diagnoses by age, sex, and location. AJR Am J Roentgenol 164:395-402

15. Kuo YL, Yao WJ, Chiu HY (2005) Role of sonography in the preoperative assessment of neurilemmoma. J Clin Ultrasound 33:87-89

16. Kuo YL, Chiu HY, Yao WJ, Shieh SJ (2009) Ultrasound for schwannoma in the upper extremity. J Hand Surg [Eu] 34:697-698

17. Oberle J, Kahamba J, Richter HP (1997) Peripheral nerve schwannomas-an analysis of 16 patients. Acta Neurochir (Wein) 139:949-953

18. Ozdemir O, Ozsoy MH, Kurt C, Coskunol E, Calli I (2005) Schwannomas of the hand and wrist: long-term results and review of the literature. J Orthop Surg (Hong Kong) 13:267-272

19. Park MJ, Seo KN, Kang HJ (2009) Neurological deficit after surgical enucleation of schwannomasof the upper limb. J Bone Joint Surg Br 91:1482-1486

20. Patel MR, Mody K, Moradia VJ (1996) Multiple schwannomas of the ulnar nerve: a case report. J Hand Surg [Am] 21:875-876

21. Phalen GS (1976) Neurilemmomas of the forearm and hand. Clin Orthop Relat Res 114:219-222

22. Saito S, Suzuki Y (2010) Schwannomatosis affecting all three major nerves in the same upper extremity. J Hand Surg [Eu] 35:592-594
23. Sandberg K, Nilsson J, Søe Nielsen N, Dahlin LB (2009) Tumours of peripheral nerves in the upper extremity: a $22-$ year epidemiological study. Scand J Plast Reconstr Surg Hand Surg 43:43-49

24. Sawada T, Sano M, Ogihara H, Omura T, Miura K, Nagano A (2006) The relationship between pre-operative symptoms, operative findings and postoperative complications in schwannomas. J Hand Surg [Br] 31:629-634

25. Strickland JW, Steichen JB (1977) Nerve tumors of the hand and forearm. J Hand Surg [Am] 2:285-291

26. Rockwell GM, Thoma A, Salama S (2003) Schwannoma of the hand and wrist. Plast Reconstr Surg 3:1227-1232

27. Takase K, Yamamoto K, Imakiire A (2004) Clinical pathology and therapeutic results of neurilemmoma in the upper extremity. J Orthop Surg 12:222-225

28. Tanabe K, Tada K, Ninomiya H (1997) Multiple schwannomas in the radial nerve. J Hand Surg [Br] 22:664-668

29. Tang JB, Ishii S, Usui M, Naito T (1990) Multifocal neurilemomas in different nerves of the same upper extremity. J Hand Surg [Am] 15: 788-792

30. Vlychou MI, Dailiana ZH (2011) Ancient schwannoma of the hand. J Hand Surg [Am] 36:2030-2033 